\title{
Práctica exclusiva de crol frente a práctica de los cuatro estilos de nado en el perfeccionamiento de la técnica de crol Exclusive practice of crawl versus practicing the four swimming strokes on the improvement of crawl technique \\ *Pablo Prieto González, **Jaromir Sedlacek \\ *Prince Sultan U niversity (Saudi Arabia), **U niversity of Prešov (Slovakia)
}

\begin{abstract}
Resumen. Contexto: Existen muchos aspectos relativos al proceso de enseñanza-aprendizaje en natación que no han sido objeto de estudio. O bjetivo: El propósito de la presente investigación fue verificar si el perfeccionamiento de la técnica de crol es más eficaz cuando se practica crol de forma exclusiva, o cuando se realizan sesiones de los cuatro estilos. Método: 26 estudiantes universitarios de sexo masculino fueron aleatoriamente asignados a dos grupos: Grupo crol (GC): Realizó exclusivamente sesiones de crol. Grupo estilos (GE): Realizó sesiones de los cuatro estilos de nado. Resultados: Una vez finalizado el programadeintervención de ocho semanas, ambos grupos mejoraron latécnica decrol. Sin embargo, las mejoras obtenidas por el GE fueron significativamente superiores a las logradas por el GC en el test de $25 \mathrm{~m}$ crol ( $p=.001$ ), en la longitud de ciclo $(p=.006)$, en la velocidad de nado promedio $(p<.001)$, y en el índice de nado $(p=.002)$. Conclusión: El perfeccionamiento de latécnicade crol en varones en edad universitariaes más eficaz si se realizan sesiones delos cuatro estilos de nado, que si se practica exclusivamente crol.
\end{abstract}

Palabras clave: N atación, técnica, principio de variedad, principio de especificidad.

\begin{abstract}
Context: Many aspects related to the teaching-learning process in swimming have not been subject of study. 0 bjective: The purpose of the present researchwas to verify which methodological approach is more effective in improving the crawl technique: Exclusive practice of crawl, or practicing the four swimming strokes. Method: 26 male university students were randomly allocated into two groups: Crawl Group (GC):They exclusively performed crawl sessions. Swimming strokes group (GE): They underwent sessions of all the four swimming strokes. Results: After completing an eight-week intervention program, both groups enhanced their crawl technique. However, improvements observed in the GE were significantly greater than those achieved by the GC in the 25 -metres cravl test $(p<.001)$, stroke length $(p=.006)$, average swimming speed $(p<.000)$, and stroke index ( $p=.002)$. Conclusion: Practicing all four swimming strokes is more effective than crawl swimming only when it comes to improving the crawl technique in college-aged males.
\end{abstract}

Key words: Swimming, technique, principle of variety, principle of specialization.

\section{Introducción}

La enseñanza-aprendizaje de las técnicas básicas y específicas de la natación es un proceso que debe ser convenientemente planificado y evaluado (Morales, 2015). Para ello se han de tener en cuentalos siguientes aspectos: Las características de los alumnos o nadadores, las características de la natación, los aspectos biomecánicos de la técnica de nado, los objetivos que se pretenden conseguir, y los planteamientos pedagógicos 0 métodos didácticos que se han de emplear en cada caso (Huaycha \& Prado, 2015).

Tradicionalmente, la enseñanza de la técnica en natación comenzaba con la realización de actividades de familiarización y el aprendizaje de las técnicas básicas

Fecharecepción: 02-12-19. Fechadeaceptación: 02-11-20

Pablo Prieto González

pabloccjb@gmail.com propias del medio acuático: Flotación, respiración, propulsión, equilibrio y manipulación de objetos. A continuación, se procedía a la enseñanza de los cuatro estilos: Crol, espal da, braza y mariposa.Y finalmente, se practicaban otras actividades deportivas acuáticas: Water polo, saltos y sincronizada (Barbosa \& Q ueirós, 2004). La estrategia en la práctica más utilizada era la analítica progresiva, en combinación con la estrategia global. Esta metodología está inspirada en el alto rendimiento deportivo (M oreno, 1998).

Posteriormente, estos planteamientos han sido cuestionados, y se han llevado a cabo estudios en los que se ha tratado de contrastar la eficacia de las diferentes metodologías existentes (Bovi, 2006). Estas investiga ciones han aportado información acerca de la metodología más eficaz de aprendizaje en natación en la etapa infantil (Saldias, González, Caro, Guzmán, Jerez, \& Lobos, 2019), la conveniencia de utilizar material flotante (Serrano \& Ferriz, 2018), y las ventajas de reali- 
zar las sesiones en piscinas superficiales o profundas (Rocha, Marinho, Garrido, M orgado \& Costa, 2018). Pero la mayor parte de estos estudios se han centrado en la infancia y en el nivel de iniciación.

Por tanto, existen numerosos aspectos relativos al proceso de enseñanza-aprendizaje en natación que no han sido objeto de estudio. De hecho, en el caso de los adultos, se desconoce cuál es el mejor planteamiento metodológico para el aprendizaje y perfeccionamiento de los estilos de nado (Serrano \& Ferriz, 2018). Varios autores recomiendan comenzar con la enseñanza de la técnica de crol o de espalda en el caso de los niños, mientras que en adultos proponen empezar con braza o espalda, debido a que la respiración se puede realizar con mayor facilidad (Serrano \& Ferriz, 2018).

Con respecto a los adultos, también se desconoce si el aprendizaje y el perfeccionamiento de los estilos de nado se ha de realizar de forma conjunta o separada (Rubio \& Zhañay, 2012), y si la mejora de la técnica de un estilo concreto es más eficaz cuando se practican los cuatro estilos o uno solo. Es decir, si sería más conveniente aplicar el principio de variabilidad de la práctica 0 el de especificidad.

En este sentido cabe indicar que, tradicionalmente, se ha considerado que el aprendizaje motor es más eficaz cuando la práctica se lleva a cabo en condiciones de especificidad (Seidler, 2010). Sin embargo, diversos autores entienden que la variabilidad de la práctica mejora en mayor medida el aprendizaje motor. Sostienen que la variabilidad facilita la transferencia entre ta reas similares, favorece la formación de un esquema motor que permite adaptar el movimiento a situaciones cambiantes, y mejora el procesamiento de la información (Caramiaux, Bevilacqua, Wanderley $\&$ Palmer, 2018; Chua, Dimapilis, Iwatsuki, Abdollahipour, Lewthwaite \& Wulf, 2019).

Numerosos estudios han tratado de analizar el efecto la variabilidad de la práctica en el aprendizaje motor (Caballero, Moreno, Reina, Roldán, Coves, \& Barbado 2017). Y la realidad es que se han encontrado resultados contradictorios, puesto que, en algunas ocasiones, una mayor variabilidad resultó positiva (Chua et al., 2019; Seidler, 2010; Shea \& Kohl, 1990), y en otros casos fue negativa (Antúnez, Hernández, García, Vállo, Arroyo, 2012; U rbán, Hernández-Davó \& M oreno, 2012). Estas discrepancias podrían deberse a que la variabilidad de la práctica podría ejercer un efecto positivo en el aprendizaje motor dependiendo de factores como: El tipo de actividad física o deportiva (tareas abiertas o cerradas), el dominio de la tarea que se pretenda mejorar, y las capacidades del sujeto (Caballero et al., 2017; StarkInbar, Raza, Taylor \& Ivry, 2017).

En el caso concreto de la natación, existen autores que apuestan por incrementar la variabilidad de la práctica para mejorar los comportamientos adaptativos del nadador (Guignard, Rouard, Chollet \& Seifert, 2017), y para desarrollar el sentido del agua (Magias \& Pill, 2013, pp. 93-101). Sin embargo, en ningún estudio seha analizado si la mejora de la técnica de un estilo de nado es más eficaz cuando se practican los cuatro estilos, 0 cuando se practica exclusivamente el estilo concreto que se pretende mejorar.

En este contexto, el objetivo del presente estudio fue determinar si la mejora de la técnica de crol en adultos en edad universitaria es más eficaz practicando exclusivamente crol, o real izando sesiones de los cuatro estilos.

\section{Método}

Se realizó un estudio de experimental de tipo longitudinal.

\section{Sujetos}

26 estudiantes universitarios de sexo masculino ma triculados en la asignatura Beginner Swimming de la Universidad Prince Sultan (Riad, Arabia Saudita), participaron voluntariamente en el presente estudio. A fin de garantizar que no padecían ninguna lesión o enfermedad que les impidiese realizar con normalidad las actividades y los test llevados a cabo durante la investiga ción, cumplimentaron el Cuestionario de Aptitud para laActividad Física, conocido como PAR-Q test (Thomas, Reading \& Shephard, 1992). Además, para ser incluido en el estudio, tuvieron que superar con éxito las siguientes pruebas de acceso: $25 \mathrm{~m}$ de nado ventral en menos de 22 seg, 25 m de nado dorsal en menos de 30 seg, 15 seg de flotación estática en posición decúbito supino y 15 seg de flotación dinámica en posición vertical. Esta batería de test es una adaptación de la propuesta de evaluación técnica en natación de Sailema, Sailema, Q uizhpe, Cabana y Gómez (2018, pp. 70-73).

N inguno de los sujetos seleccionados había practica do natación de forma regular o sistemática durante el último año. Tampoco habían acudido a clases de natación durante losúltimos cinco años. Antes del inicio del proceso de intervención, los sujetos que superaron las pruebas de acceso al estudio asistieron a dos sesiones teóricas en las que se explicaron los siguientes contenidos: Principios biomecánicos de la natación, habilidades 
motrices básicas acuáticasy aspectostécnicos de los cuatro estilos de nado (crol, braza, espalda y mariposa). También fueron debidamente informados de los objetivos del estudio y de los riesgos y beneficios derivados de su participación en el mismo. Finalmente, se les indicó que se podían retirar del estudio de forma voluntaria en cualquier momento. La investigación se realizó respetando los principios éticos recogidos en la declaración de Helsinki, y contó también con la aprobación de la Junta de Revisión Institucional del Comité de Bioética de la U niversidad Prince Sultan.

\section{Evaluación}

Las variables de tipo antropométrico (peso, estatura e IMC), fueron registradas antes de iniciarse el proceso de intervención, haciendo uso de una estación de medición Seca digital column scale (Hamburgo, Alemania). Las mediciones se realizaron con los sujetos descalzos, con una precisión de 0,1 kg parael peso, y de $0.1 \mathrm{~cm}$ para le estatura.

Por su parte, las pruebas realizadas en el pretest y en el postest se llevaron a cabo en una piscina de $25 \mathrm{~m}$. Se hizo uso de un sistema de video cronometraje compuesto por una cámara de video GoPro Hero+ $L C D$, modelo CHDHB-101 (California, USA), conectada a un ordenador portátil Toshiba Satellite C55D-C-17C (N euss, Germany). La cámara se ubicó a $10 \mathrm{~m}$ del borde lateral de la piscina, a una altura de $5 \mathrm{~m}$ sobre la superficie, y en una posición equidistante con respecto al principio y al final de la piscina. Las grabaciones de vídeo fueron analizadas con el programa Q uick time player, (version 7.7.9 for W indows, USA).

Las variables registradas para estimar las mejoras de la técnica se muestran a continuación. Todas ellas fueron de tipo cuantitativo, dado que permiten evaluar mejor y con mayor objetividad los niveles de ejecución (M orales, \& Arellano, 2005).

Test de $25 \mathrm{~m}$ crol (T25): Tiempo empleado por cada sujeto en recorrer $25 \mathrm{~m}$ nadando a crol y saliendo desde el poyete. La medición se hizo en seg.

Frecuencia de ciclo (FC): Para realizar esta medición se consideró que un ciclo de brazos comienza en el momento que una mano entra en el agua, y finaliza cuando se produce la siguiente entrada de esa misma mano en el agua (Morales y Arellano, 2005). La FC se calculó dividiendo tres ciclos entre el tiempo empleado en rea lizarlos. La medición se realizó inmediatamente después de que la cabeza del sujeto superase una marca situada a $10 \mathrm{~m}$ de la salida. El valor obtenido se expresó en hercios(Hz) (Washino, Mayfield, Lichtwark, M ankyu
\&Yoshitake, 2019).

Longitud de ciclo (LC): Esla distancia que el cuerpo del nadador recorre con cada ciclo de brazos. Se midió en $m$, y se calculó utilizando la siguiente fórmula: $\mathrm{LC}=\mathrm{VNp} / \mathrm{FC}$.

Velocidad de nado promedio (VN): Para su medición, se hizo uso de dos marcas, unasituadaa 10 y otraa $20 \mathrm{~m}$ de la línea salida, y se registró el tiempo transcurrido entre el paso de la cabeza del sujeto por la primera y por la segunda marca. Posteriormente, la VN se calculó mediante la siguiente fórmula: $V=E / T$. El valor obtenido se expresó en $\mathrm{m} / \mathrm{seg}$

Índicede nado (IN ): Se cal culó multiplicando la velocidad promedio por la longitud de ciclo promedio, mediante la siguiente fórmula: IN $=$ VN $*$ LC. El valor obtenido se expresó en $\mathrm{m}^{2}$ / s*ciclo (G anzevles, Vullings, Beek, Daanen, \& Truijens, 2017).

Diminuciones del T25, e incrementos en la LC, la VN y el IN fueron interpretados como mejoras de la ejecución técnica. La variable FC fue incluida también en el estudio debido a que es uno de los dos factores que condiciona el IN (García, 2016; Sánchez \& Arellano, 2002).

\section{Procedimiento}

Los 26 sujetos fueron aleatoriamente divididos en dos grupos experimentales: Grupo crol (GC: Edad: $20.16 \pm 1.17$ años; peso: $74.4 \pm 7.4$ kg; estatura: 177.5 $\pm 9.5 \mathrm{~cm}$; IMC: $23.6 \pm 3.9$ ) y grupo estilos (GE: Edad: $20.39 \pm 1.09$ años, peso: $75.2 \pm 6.3$ kg; estatura: 177.2 $\pm 9.1 \mathrm{~cm}$; IMC: $24.1 \pm 2.2$ ). El GC realizó exclusiva mente actividades y ejercicios de perfeccionamiento de la técnica de crol durante todo el período de intervención (16 sesiones). Por el contrario, en el GE, las cuatro primeras sesiones se dedicaron al perfeccionamiento de la técnica de crol, las cuatro siguientes a la técnica de espalda, las cuatro siguientes a la mejora de la técnica de braza, y las cuatro últimas al perfeccionamiento de la técnica de mariposa. El proceso de intervención utilizado se detalla en la tabla 1. Las sesiones del mismo aplicadas tanto al GC como al GE fueron diseñadas y llevadas a cabo por la misma persona: Un Licenciado en Educación Física y Entrenador Superior de Natación, con más de 20 años de experiencia en la enseñanza de este deporte.

\section{Análisis estadístico}

El análisis estadístico se realizó con el programa informático IBM SPSSV. $22 ®$. Los resultados se presentan usando el siguiente formato: M edia aritmética (des- 
Tabla 1

Características del proceso de intervención.

Duración del proceso de 8 semanas. Se realizaron dos sesiones semanales, (lunes y miércoles) con una duración de 50 min

intervención

Objetivos del proceso de $\quad$ GC: Perfeccionamiento de la técnica de crol mediante:

- La reducción de la resistencia de formay de oleaje

-La mejora de la continuidad, fluidez y precisión de las acciones propulsivas

- La estabilización de las repeticiones exitosas

- La corrección de errores técnicos

-La reducción del costo energético

GE: Ídem para los estilos de crol, espalda, braza y mariposa

Aspectos metodológicos El proceso de enseñanza aprendizaje se diseñó partiendo del nivel de los participantes. Las explicaciones fueron claras y breves para favorecer la asimilación de las indicaciones asegurándose en todo momento que comprendían las tareas y actividades que tenían que realizar. La transmisión de información se efectuó haciendo uso del canal auditivo, visual y kinestésico. Asimismo, para evitar la acumulación de fatiga y el consiguiente deterioro en la ejecución técnica durante la realización de los ejercicios, se utilizaron pausas de recuperación adecuadas recuperación adecuad sa

$\begin{array}{ll}\text { Estilos de nado } & \text { GC: Crol } \\ & \text { GE: Crol, espalda, brazay mariposa }\end{array}$

Estilos de enseñanza utilizados A signación de tareas, enseñanza recíproca, grupos reducidos y descubrimiento guiado (Fernández \& Espada, 2015)

Estrategias en la practica Analítica progresiva, global con modificación de la situación real, global con polarización de la atención y gl obal pura (Delgado-Noguera, 1993, pp. 1045-1066).

Tipo de ejercicios utilizados La estructura de las sesiones utilizadas con el GC y el GE fue idéntica, y los ejercicios realizados equiparables. En ambos grupos experimentales se emplearon siete tipos de ejercicios, tal y como se detall a a continuación:

-Ejercicios dirigidos a la mejora del batido. Ejemplo GC: Realizar el batido de crol sentado en el borde de la piscina, y posteriormente con tabla. Ejemplo GE: Igual que el anterior, pero para los cuatro estilos

-Ejercicios dirigidos a la mejora del barrido. Ejemplo GC: Realizar el barrido sin movimiento de piernas, colocando un pull-boy entre ambos muslos. Ejemplo GE: Igual que el anterior, pero para los cuatro estilos

-Ejercicios de coordinación: Ejemplo GC: Nadar a crol, realizando el batido de mariposa. Ejemplo GE: Nadar a crol, braza o espalda efectuando el batido de mariposa -Ejercicios de contraste. Ejemplo GC: Nadar a crol con la mano cerrada, a continuación, los dedos separados y extendidos, y finalmente con los dedos juntos y extendidos. Ejemplo GE: Igual que el anterior, pero para los cuatro estilos

-Ejercicios de sustentación. Ejemplo: Haciendo el batido de crol (GC) o de crol, braza, espal da o mariposa (GE), avanzar realizando remadas de sincronizada con ambas manos -Ejercicios de propulsión. Ejemplo: Realizar el batido de crol (GC) o de crol, braza, espalda o mariposa (GE) mientras que con los brazos se efectúa la patada de pato con recobro bajo el agua

-Ejercicios para la disminución de la resistencia al avance: Después de nadar $15 \mathrm{~m}$ a gran velocidad a crol (GC) 0 a crol, braza, espal da o mariposa (GE), detenerse adoptando la posición de flecha, tratando de llegar al final de la piscina

-Ejercicios de corrección y sobrecompensación de errores. Ejemplo para GC: Si la entrada de la mano en el agua se produce por dentro de la línea central de cuerpo, pedir a sujeto que la haga por fuera de dicha línea. Ejemplo para GE: El sujeto realiza un rolido reducido en el nado de espalda. Se pide al sujeto que nade pegado a la corchera y realice un rolido superior a $90^{\circ}$

Feedback El feedback proporcionado fue tanto de tipo verbal como no verbal (visual y kinestésico) y preferentemente individualizado, tratando de explicar en la medida de lo posible las relaciones causa-efecto de cada acción. Los sujetos recibieron infor mación relativa a la ejecución de las acciones técnicas y al resultado de las mismas. El feedback ofrecido fue además de tipo positivo y de tipo correctivo, manteniendo una ratio $2 / 1$ entre el primero y el segundo para favorecer la motivación de los participantes \begin{tabular}{ll}
$\begin{array}{ll}\text { Recur sos materiales e } \\
\text { instal aciones }\end{array}$ & Las sesiones se llevaron a cabo en una piscina de $25 \times 12.5 \mathrm{~m}$, y de profundidad mixta (de 1.2 a $3.5 \mathrm{~m}$ ). El agua se encontraba a una temperatura de $260 \mathrm{C}$. Se utilizaron tanto pull- \\
\hline
\end{tabular}

viación típica). La normalidad de los datos fue contrastada mediante la prueba de Shapiro-W ilk, y la homogeneidad delasvarianzas por medio de lapruebade Levene. Para verificar que no existían diferencias significativas entre el GC y el GE en ninguna de las variables registradas en el pretest, se utilizó la prueba t de Student para muestras independientes. Por su parte, para valorar las diferencias entre el pretest y el postest dentro de cada grupo, el porcentaje de cambio se calculó a tra vés de la fórmula \% cambio $=([$ postest - pretest $] /$ pretest $*$ 100), lasignificanciaestadística con la pruebat de Student para muestras relacionadas y el tamaño de efecto con la d de Cohen. Los valores obtenidos en esta última prueba fueron interpretados del siguiente modo: $d<0.19$, insignificante; $0.20 \leq d \leq 0.49$, pequeño; $0.5 \leq d \leq$ 0.79 medio; $d>0.8$ grande (N akagawa $\&$ Cuthill, 2007). La comparación entre grupos se realizó mediante un ANOVA de medidas repetidas (grupo $x$ tiempo). $Y$ en caso de encontrar efectos significativos, se aplicó el test post hoc de Tukey. El nivel de significación establecido fue de $p<.005$.

\section{Resultados}

La prueba t de Student para muestras independientes reveló que no existían diferencias significativas en el pretest entre el GC y el GE en ninguna de las variables analizadas: T25: $p=.55 ; \mathrm{FC}: \mathrm{p}=.54 ; \mathrm{LC}: \mathrm{p}=.89 ; \mathrm{VN}: \mathrm{p}$ $=.58 ; \mathrm{IN}: p=.71$. Con respecto a las diferencias entre el pretest y el postest dentro de cada grupo, el GC obtuvo mejoras significativas en el T25, en la FC y en la VN, siendo los tamaños de efecto grandes en los tres casos. En cambio, en este mismo grupo, no se observaron mejoras significativas ni en la LC ni en el IN. Por su parte, en el GE se registraron mejoras significativas en todos los parámetros evaluados (T25, FC, LC, VN e IN), siendo también los tamaños de efecto grandes (ver tabla 2).

En cuanto a las diferencias entre ambos grupos, el ANOVA reflejó que los resultados obtenidos por el GE fueron significativamente superiores a los logrados por el GC en todas las variables analizadas excepto en la $F C$, tal y como se detalla a continuación:T25: $p=.001$; $F C: p=.782 ; L C: p=.006 ; V N: p<.001 ; I N: p=0.002$.

Tabla 2

Cambios obtenidos por el GC y el GE tras la aplicación del proceso de intervención

\begin{tabular}{|c|c|c|c|c|c|c|}
\hline \\
\hline & Pretest & Postest & $p$ & (Cohen's d) & \% Cambio & [IC95\%] \\
\hline T25 (seg) & 18.95 (1.37) & $18.37(1.46)$ & .006 & -0.98 & -3.06 & {$[0.11,1.04]$} \\
\hline & & & .006 & 1 & & \\
\hline & & & .597 & 0. & 0. & \\
\hline VN $(\mathrm{n}$ & 1.2 & 1.26 ( & .010 & 0.9 & 3.2 & \\
\hline IN $(\mathrm{m}$ & & 1.8 & .113 & 0.5 & 4.02 & \\
\hline \multicolumn{7}{|l|}{$\overline{\mathrm{GE}}$} \\
\hline & Pretest & Postest & $p$ & (Cohen's d) & \% Cambio & [IC95\%] \\
\hline & $18.62(1.42)$ & $17.17(0.94)$ & .000 & -4.13 & -7.78 & {$[1.02,1.88$} \\
\hline & 08710031 & 0.891 & .001 & 1.5 & 2.29 & {$[-0.09,0.0$} \\
\hline in & & 1.5 & .001 & 1. & 5.5 & {$[-0.2]$} \\
\hline VN ( & $24(0.09)$ & $1.34(0.07)$ & .000 & 4.08 & 8.06 & {$[-0.21,0.0]$} \\
\hline IN (m²/s*ciclo) & $1.78(0.32)$ & $2.03(0.28)$ & .000 & 2.88 & 14.04 & {$[-0.46,0.04$} \\
\hline
\end{tabular}




\section{Discusión}

En el presente estudio se ha podido verificar que, tanto el grupo que practicó crol de forma exclusiva, como el grupo que realizó sesiones de los cuatro estilos de nado consiguieron mejorar la técnica de crol. Pero también se ha constatado que la última de las dos intervenciones generó mejores resultados en el T25, en la LC, en laVN y en el IN. En el caso de la FC, es posible que no se hayan producido diferencias significativas entre ambos grupos debido a que este parámetro mejora a través de la realización ejercicios de velocidad asisti$d a$, y dichas actividades no se incluyeron en las sesiones de nado llevadas a cabo.

Aun así, los resultados sugieren que el trabajo bra za, mariposa y especialmente el de espalda podría ha ber generado un impacto positivo en la técnica de crol, de modo que cabría la posibilidad de que dentro del GE se hayan producido transferencias a nivel cognitivo y motriz esenciales para la mejora de la técnica de nado (Lawton, 2013, p.10). Es decir, transferencias de elementos que han podido contribuir a mejorar aspectos tales como: La comprensión de principios básicos de la natación, la adopción de posiciones propulsivas, la disminución de la resistencia al avance, el desarrollo del sentido del agua y la coordinación entre ambos brazos, entre el barrido y la respiración y entre miembros superiores e inferiores. Sin embargo, dado que en la actualidad no existe ningún estudio que haya anal izado en profundidad las interacciones que se producen entre los diferentes estilos de nado, ni que aspectos técnicos son susceptibles de ser transferidos entre un estilo y otro, es preciso que todas estas cuestiones sean aclaradas en futuras investigaciones.

Pero al margen de las posibles transferencias entre estilos, otro factor que podría explicar las diferencias en favor del GE es de tipo psicológico. Es razonable pensar que realizar ejercicios de los cuatro estil os de nado puede generar un mayor nivel de motivación que la práctica exclusiva de crol, debido a la variedad y diversidad de la práctica (O rtega, 2006, p. 59).

En virtud de los resultados del estudio, y de las diferencias entre ambos grupos en favor del GE, también cabría interpretar que el principio de variedad es más relevante que el principio de especificidad en el perfeccionamiento de la técnica de crol. En este sentido es preciso recordar que, el principio de variedad, que está inspirado en la teoría del esquema de Schmidt (Canode-la-Cuerda, M olero-Sánchez, Car rataláTejada, Algua cil-Diego, Molina-Rueda, Miangolarra-Page, \&
Torricelli, 2015; M orales, 2016), se aplica preferentemente en la iniciación deportiva, en etapas infantiles, y sus defensores consideran además que es de gran utilidad en tareas con un cierto componente perceptivo y decisional (Hernández \& Valero, 2019). Sin embargo, en este estudio, la utilización del principio de variedad en el perfeccionamiento de la técnica de crol fue eficaz en un contexto de enseñanza-aprendizaje en el que es más habitual aplicar el principio de especificidad. Por tanto, de cara a futuras investigaciones, se debería tener en cuenta que la variabilidad de la práctica puede ser útil no solo en el aprendizaje de la técnica, sino no también en el perfeccionamiento; no solo en etapas infantiles, sino también con adultos; y no solo en deportes de adversario o colectivos, sino también en un deporte individual con mínimas o nulas exigencias a nivel táctico.

Ahora bien, con respecto al principio de variedad, es necesario tener presente que su aplicación conlleva un cierto grado de dificultad. En este sentido, Balagué, Torrents, Pol y Seirul-lo (2014) señalan que las varia ciones no pueden ser aleatorias, ni regulares, ni tampoco pequeñas, porque podrían no generar cambios coordinativos. Pero también indican que no pueden ser excesivas, dado que podrían dificultar la integración de sinergias. Por lo tanto, en el caso del presente estudio, y atenor de las notables mejoras logradas por el GE, cabe pensar que los ejercicios de los estilos de espalda, braza y mariposa supondrían con respecto al estilo de crol un nivel de variabilidad de la práctica adecuado para favorecer el perfeccionamiento de la técnica de crol.

Por otra parte, es preciso aclarar también que la aplicación del principio de variedad no significa en ningún caso que el principio de especificidad carezca de utilidad en el contexto deportivo, sino todo lo contra rio, dado que tiene una gran importancia cuando se tra ta de atletas entrenados (Tanaka, 1994). Lo que ocurre es que el uso del principio de especificidad debe estar precedido de una apropiada aplicación del principio de multilateralidad (Carrera\& Bompa, 2007, p. 26). De lo contrario, el entrenamiento puede generar problemas detipo físico o psicológico, y provocar el temprano abandono de la práctica deportiva (Rego-Neira, NavarroPatón \& A billeira-González, 2014).

En el caso de la natación, tradicionalmente se han aplicado metodologías extrádas del ámbito competitivo al educativo. La enseñanza estaba centrada en los conocimientos que se deberían transmitir, que eran organizados de forma secuencial (Pla-Campàs, 2015). En cambio, las investigaciones en el ámbito educativo han 
subrayado la necesidad de que el sujeto sea el protagonista de su proceso de enseñanza-aprendizaje (Romero, López, Ramírez, Pérez \& Tejada, 2008). En esta misma línea, Cecchini, Fernández, Pallasá y Cecchini (2012) cuestionan también el proceso de aprendizaje secuencial y progresivo. En su lugar proponen que las actividades de enseñanza-aprendizaje sean variadas, y no se jerarquicen en función de su dificultad, pues entienden que el aprendizaje se produce en paral elo y no de forma seriada.

En consecuencia, bajo estas premisas, y en virtud también de los resultados del presente estudio, se ha de tener en cuenta que el perfeccionamiento de la técnica en natación se puede ver dificultado por la secuenciación de los estilos de nado en función de su nivel de dificultad, y por la no aplicación del principio de variabilidad de la práctica. En cambio, en el caso del crol, practicar los cuatro estilos en períodos de tiempo relativamente breves podría favorecer las mejoras a nivel técnico. De hecho, realizar una práctica más variada permitirá a los alumnos o nadadores elaborar su estilo propio, que estará además adaptado a sus características biológicas y a sus circunstancias personales, aunque para ello se ha de contar también con la participación activa y consciente del sujeto (Balagué et al., 2014).

Para finalizar, es preciso hacer mención a las limitaciones del estudio. En primer lugar, es necesario indicar que habría sido deseable haber realizado una evaluación de la técnica de nado mediante el análisis de las imágenes obtenidas a través de la filmación subacuática de los sujetos. Asimismo, también se debe destacar que, en la presente investigación, se han analizado las mejoras que genera la práctica de los cuatro estilos de nado en la técnica de crol. Sin embargo, son necesarias más investigaciones en las que se examine el efecto de diferentes niveles y cargas de variabilidad de la práctica en el perfeccionamiento de la técnica en natación (Dhawale, Smith \& Ö Iveczky, 2017). De este modo, se podrá obtener información más precisa para la creación de transferencias positivas entre los diferentes estilos de nado (Gómez, 2012).

\section{Conclusión}

El perfeccionamiento de la técnica de crol en varones en edad universitaria es más eficaz cuando se realizan sesiones de los cuatro estilos de nado, que cuando se practica crol de forma exclusiva.

\section{Referencias}

Antúnez, R.M., Hernández, F.J., García, J.P.,Vaillo, R.R., \&Arro- yo, J.S. (2012). Relationship between motor variability, accuracy, and ball speed in the tennis serve. Journal of H uman Kinetics, 33, 45-53.

Balagué, N., Torrents, C. , Pol, R. \& Seirul-lo, F. (2014). Entrena miento integrado. Principios dinámicosy aplicaciones Apunts, $116(2), 60-68$.

Barbosa, T.M., \& Q ueirós, T.M.G. (2004). Ensino da natação: U ma perspectiva metodológica para a abordagem das habilidades motoras aquáticas básicas. Lisboa: Xistarca.

Bovi, F. (2006). Análisis de la enseñanza en natación. Evaluación y contraste de los métodos sistemático y lúdico (Tesis doctoral inédita). Universidad de LasPalmasde GranCanaria, LasPalmasde GranCanaria.

Caballero, C., Moreno, F.J., Reina, R., Roldán, A., Coves, A., \& Barbado, D. (2017). The role of motor variability in motor control and learning depends on the nature of thetask and the individual's capabilities. European Journal of Human M ovement, $38,12-26$.

Cano-delaCuerda, R., Molero-Sánchez,A., Carratalá Tejada, M., Alguacil-Diego, I.M., M olinaRueda, F., MiangolarraPage, J.C., \& Torricelli, D. (2016). Teorías y modelos de control y aprendizaje motor. Aplicaciones clínicas en neurorrehabilitación. Neurología, 30 (1), 32-41.

Caramiaux, B., Bevilacqua, F., Wanderley, M.M., \& Palmer, C. (2018) Dissociable effects of practice variability on learning motor and timing skills. PLoS On, 13(3), e0193580.

Carrera, M ., \& Bompa, T. (2007). Theory and methodology of training: General perspectives, En B. Blumenstein, R. Lidor, \& G. Tenenbaum (Eds), Physiology of sport training.Vol. 2 (p.26). 0 xford: Mayer \& M ayer Sport.

Cecchini,J.A., Fernández, J.L., Pallasá, M. , \& Cecchini, C. (2012). El proceso detransferenciaen el aprendizajey desarrollo motor. Psicothema, 24(2), 205-210.

Chua, L.-K., Dimapilis, M.K., Iwatsuki, T., Abdollahipour, R., Lewthwaite, R., \& Wulf, G. (2019). Practice variability promotes an external focus of attention and enhances motor skill learning Human movement science, 64, 307-319.

Delgado-Noguera, M.A. (1993). Los métodos didácticos en la Educación Física. EnV.V.A.A. (Eds.), Fundamentos deEducación Físca para enseñanza primaria.Vol. II (pp. 1045-1066). Barcelona: Inde.

Dhawale,A.K., Smith, M.A., \&Ölveczky, B.P. (2017).TheRoleof Variability in M otor Learning Annual Review of N euroscience, 40, 479-498.

Fernández, M., \& Espada, M. (2015). Frecuenciade utilización y aceptación de estilos de enseñanza en educación física. Athlos: Revista Internacional de Ciencias Sociales de la Actividad Física, el Juego y el Deporte, 9, 44-63.

Ganzevles, S., Vullings, R. , Beek, P.J., Daanen, H., \&Truijens, M. (2017). U singTri-Axial Accelerometry in Daily Elite Swim Training Practice. Sensors, 17 (5), 990.

García, S. (2012). Índices de eficacia en la prueba de natación de 50 metros libres (Tesis de pregrado). Facultad de Ciencias de laActividad Física y del Deporte, León.

Gómez, J.M. (2012). Introducción sobre la natación en la 
Educación Física. En J.M. Gómez (Ed. ), La enseñanza de los estilos de natación: crol, espalda, mariposa y braza: M anual para monitores, profesores y practicantes (p. 15). Sevilla: Wanceulen S.L.

Guignard, B., Rouard, A., Chollet, D., \& Seifert, L. (2017). Behavioral dynamicsin swimming:The appropriate use of inertial measurement units. Frontiers of Psychology, 8, 383.

Hernández, A., \&Valero, A. (2019). M odelo para el control de los factores determinantes del rendimiento de los nadadores de 800 metros de la categoría escolar. Conrado, 15(66), 25-30.

Huaycha, E. , \& Prado, J.Y. (2015). Estrategias deenseñanza en el aprendizajede la natación en el estilo de nado crol, en estudiantes del segundo grado «a» de secundaria de la institución educativa Corazón de Jesús. (Tesis doctoral inédita). U niversidad Nacional de San Cristóbal de Huamanga. Ayacucho.

Lawton, J. (2013). Planning. En J. Lawton (Ed.), Complete Guide to Primary Swimming (p. 10). U nited States: Human kinetics.

Magias, T. \& Pill, S. (2013). Teaching swimming for movement variability: an application of teaching games for understanding-game sense. En J. Q uay y A. M ooney (Eds.), 28th ACH PER International Conference (pp. 93-101). Melbourne, Australia: Australian Council for Health, Physical Education and Recreation.

Morales, A. (2016). La variabilidad en el aprendizaje de habilidades motrices (Tesis de pregrado). Universidad Miguel Hernández, Elche, Alicante.

Morales, E. (2015). Propuesta educativa «El agua como medio de enseñanza: importanciade laeval uación». Retos, 17, 72-75.

M orales, E., \&Arellano, R. (2005). Análisis de las diferencias cuantitativas de latécnicaentre losal umnos de una escuela de enseñanza de la natación. Apunts, 79 (1), 49-58.

Moreno, J.A. (1998). ¿H acia dónde vamos en la metodología de las actividades acuáticas? Lecturas: Educación Física y Deportes. Revista Digital, 3(11). Recuperado de https:// www. efdeportes.com/ efd11/ murcia. htm

Moreno, J. A., Tella, V. \& Camero, S. (2009). Evaluación de los estilos asimétricos en grupos de edad. III Congreso Ibérico de Natación. s.d.

Nakagawa, S., \& Cuthill, I.C. (2007). Effect size, confidence interval and statistical significance: a practical guide for biologists. Biological reviews of the Cambridge Philosophical Society, 82 (4), 591-605.

Ortega, E. (2006). La participación activa como elemento principal en el proceso deformación del joven jugador de baloncesto. En E. O rtega (Ed. ), La Competición como medio formativo en el Baloncesto (p.59). Sevilla: Wanceulen S.L.

Pla-Campàs, G. (2015). Sobre mitos de la natación competitivay lanatación educativa: unamiradahistóricay cultural desde Elías Juncosa. Materiales para la H istoria del Deporte, 13, 78-95.

Rego-N eira, M., Navarro-Patón, R., \& Abilleira-González, M. (2014). Laespecial ización deportivatemprana. A proxi- mación al pensamiento de futuros maestros en educación física. Trances, 6(6), 345-356.

Rocha, H. A., Marinho, D.A., Garrido, N.D. , Morgado, L.S., $\&$ Costa, A.M. (2018). The acquisition of aquatic skills in preschool children: deep versus shallow water swimming lessons. Motricidade, 14(1), 66-72

Romero, C., López, C. . ., Ramírez,V., Pérez, A.J., \&Tejada,V. (2008). La educación física y la organización de la clase: A prendiendo a enseñar. Consideraciones previas. Publicaciones, 38, 163-182.

Rubio, M.L., \& Zhañay, C.B. (2012). Propuesta metodológica para la iniciación a la natación con niñas de los terceros de básica de la Escuela Sor Teresa Valsé. (Tesis de Pregrado). Universidad de Cuenca. Cuenca, Ecuador.

Sailema, A.A., Sailema, M., Q uizhpe, V., Cabanas, A.A., \& Gómez, A. (2018). Capítulo IV. En A.A. Sailema, M. Sailema, V. Q uizhpe, A.A. Cabanas, \& A. Gómez (Eds.), Guía metodológica para la enseñanza de la técnica de natación en la etapa de iniciación (pp. 70-72). Ecuador: Consejo Editorial U niversidadT écnica deAmbato.

Saldias, N. D., González, M., Caro, D.S., Guzmán, D.A., Jerez, S., \& Lobos, M. (2019). M etodología lúdica acuática de ambientación en niños de 6 a 10 años. Retos, 36, 336-341.

Sánchez, J.A., \& Arellano, R. (2002). Stroke index values according to level, gender, swimming style and event race distance, In: Proceedings of the XX th International Symposium on Biomechanics in Sports, K. Gianikellis, (Ed.), 56-59, Universidad de Extremadura, Cáceres.

Seidler, R.D. (2010). Neural correlates of motor learning, transfer of learning, and learning to learn. Exerciseand Sport Sciences Reviews, 38(1), 3-9.

Serrano, J. M., \& Ferriz, A. (2018). U so de material flotanteen natación. Apunts, 132(2), 48-59.

Shea, C.H. (1990). Specificity and variability of practice. Research quarterly for exercise and sport, 61(2), 169-77.

Stark-Inbar, A., Raza, M., Taylor, J.A., \& Ivry, R.B. (2017). Individual differences in implicit motor learning: task specificity in sensorimotor adaptation and sequence learning. Journal of N europhysiology, 117 (1), 412-428.

Tanaka, H. (1994). Effects of cross-training Transfer of training effects on V0 2 max between cycling, running and swimming. Sports Medicine, 18(5), 330-339.

Thomas, S., Reading, J., \& Shephard, R. J. (1992). Revision of the physical activity readiness questionnaire (PAR-Q). Canadian Journal of Sport Sciences-Revue Canadienne Des Sciences D u Sport, 17(4), 338-345.

Urbán,T.; Hernández-Davó, H. \& \& M oreno, F. J. (2012).Varia bilidad cinemática en relación con el rendimiento en el saque en jóvenes tenistas. Motricidad. European Journal of H uman M ovement, 29, 49-60.

Washino, S. , M ayfield, D. L., Lichtwark, G.A., Mankyu, H., \& Yoshitake,Y. (2019). Swimming performance is reduced by reflective markersintended for the analysis of swimming kinematics. Journal of Biomechanics, 91, 109-113. 